\title{
Levantamento fitossociológico de plantas daninhas em pomares de mangueira no semiárido mineiro
}

\author{
Flávio Henrique Silva de SENA ${ }^{1}$, Ignacio ASPIAZÚ ${ }^{1}$, Natanael Pereira da SILVA ${ }^{1}$, \\ Renato Mendes de OLIVEIRA ${ }^{1}$, Karen Marcelle de Jesus SILVA ${ }^{1}$, \\ Carlos Augusto Rodrigues MATRANGOLO ${ }^{1}$, Cleiton Fernando Barbosa BRITO ${ }^{{ }^{*}}$ \\ ${ }^{1}$ Universidade Estadual de Montes Claros- UNIMONTES, Montes Claros, MG, Brasil. \\ E-mail: cleiton.cfbb01@yahoo.com.br
}

\begin{abstract}
Recebido em agosto/2018; Aceito em novembro/2018.
RESUMO: Objetivou-se identificar as principais espécies de plantas daninhas em cultivo de mangueira aos dois e oito anos após plantio. $\mathrm{O}$ estudo foi desenvolvido em áreas irrigados de manga 'Palmer' localizadas em Janaúba-MG. Para estudo fitossociológico das plantas daninhas foi utilizado o método quadrado inventário $\left(0,25 \mathrm{~m}^{2}\right)$. Após classificação e quantificação das espécies, foi calculado calcularam-se a frequência $(\mathrm{F})$, frequência relativa $(\mathrm{Fr})$, densidade $(\mathrm{D})$, densidade relativa $(\mathrm{Dr})$, abundância $(\mathrm{A})$, abundância relativa (Ar), índice de valor de importância (IVI), índice de valor de importância relativa (IVIr) e índice de similaridade. Na área com cultivo de mangueira em produção, após oito anos do plantio, foram encontradas 17 espécies, 14 gêneros e oito famílias, sendo Sida rhombifolia a principal espécie com IVIr de 27,8\%. Já na área com dois anos de plantio encontrou-se menos espécies com destaque para Paspalum urvillei com IVIr de 70,61\%. O índice de similaridade entre as duas áreas foi de 18,18\%. Portanto, conclui-se que nas condições locais do cultivo de mangueira de Janaúba-MG, $P$. urvillei e $S$. rhombifolia são as principais espécies encontradas em pomares após dois e oito anos após implantação, respectivamente, e além disso, estes apresentam diferentes espécies infestantes comprovado pelo valor do índice de similaridade.
\end{abstract}

Palavras-chave: fitossociologia; plantas daninhas; Mangifera indica.

\section{Weed phytosociological survey on mango orchards in the minas gerais semiarid}

\begin{abstract}
This study aimed to identify the main weed species in mango cultivation at two and eight years after planting. The study was developed in irrigated areas of 'Palmer' mango located in Janaúba-MG. For weed phytosociological study, the square inventory method $\left(0.25 \mathrm{~m}^{2}\right)$ was used. The frequency $(\mathrm{F})$, relative frequency (Rf), density (D), relative density (Rd), abundance (A), relative abundance (Ra), value index of importance (VII), relative importance value index (RIVI) and similarity index were determined. In the area with mango cultivation in production, after eight years of planting, 17 species, 14 genera and eight families were found, being Sida rhombifolia the main species, with RIVI of $27.8 \%$. In the area with two years of cultivation were found less species, with emphasis to Paspalum urvillei, with RIVI of $70.61 \%$. The similarity index between the two areas was $18.18 \%$. Therefore, it is concluded that in the local conditions of the cultivation of mango of Janaúba-MG, P. urvillei and S. rhombifolia are the main species found in orchards after two and eight years after implantation, respectively, and, in addition, both present different weed species, as proven by the low similarity index value.
\end{abstract}

Keywords: phytosociology; weed; Mangifera indica.

\section{INTRODUÇÃO}

O Brasil possui uma área plantada de manga (Mangifera indica L.) de 61.800 hectares, produção de 1.002 .200 toneladas e produtividade média de $16.206 \mathrm{~kg} \mathrm{ha}^{-1}$. Entre os estados, Minas Gerais é o quarto maior produtor com 5,5 mil hectares plantadas, produção de 85.700 toneladas e produtividade $15.671 \mathrm{~kg} \mathrm{ha}^{-1}$, sendo que se destacam os municípios localizados na região Norte de Minas, principalmente, Jánaúba e Matias Cardoso, com participação de $26 \%$ da produção do Estado e apresentam produtividades (25.000 e $20.000 \mathrm{~kg} \mathrm{ha}^{-1}$, respectivamente) superiores a media nacional e do estado (IBGE, 2016).

A região norte de Minas Gerais é caracterizada por clima semiárido e apresenta condições de alta luminosidade, baixa umidade relativa e elevada temperatura que associados ao uso adequado da irrigação favorecem o cultivo de manga com obtenção de alta produtividade e qualidade dos frutos (SANTOS et al. 2016; COTRIM et al., 2017).

Existem diversos fatores que influenciam na redução da produção da mangueira e, assim, para contribuir com o manejo ideal da cultura foram realizadas pesquisas, principalmente, em relação à adubação (SILVA et al., 2013; MEDEIROS et al., 2014; CARNEIRO et al., 2017), podas e indução floral (OLIVEIRA et al., 2015; OLIVEIRA et al., 2017), irrigação (SANTOS et al. 2016; COTRIM et al., 2017; WEI et al., 2017) e manejo de pragas e doenças (SARWAR et al., 2014; SHARMA et al., 2013). No entanto, a infestação de plantas daninhas, que é outro fator limitante da produtividade para a maioria das culturas, ainda é pouco estudada no contexto produtivo da mangueira. 
As plantas daninhas exigem para seu desenvolvimento os mesmos fatores exigidos pela cultura de interesse, ou seja, água, luz, nutrientes e espaço, o que estabelece um processo de competição quando ambas se desenvolvem em um mesmo local e em determinado período do ciclo vegetativo (VASCONCELOS et al., 2012). Assim, infere-se que a infestação de plantas daninhas pode afetar diretamente a produtividade na cultura da mangueira, dificultar os tratos culturais e, consequentemente, diminuir a renda do produtor.

Para evitar ou diminuir convivência dessas plantas com a cultura da mangueira torna-se necessário à utilização do manejo correto e, para isso, deve-se inicialmente identificar as principais espécies infestantes. Assim, devem-se realizar estudos fitossociológicos que são importantes para analisar o impacto que os sistemas de manejo e as práticas agrícolas exercem sobre a dinâmica de crescimento e ocupação de comunidades daninhas em sistemas produtivos (PITELLI, 2000).

Os estudos fitossociológicos classificam, quantificam e comparam as populações de plantas daninhas num determinado momento, logo se faz necessário realizar esses estudos em diferentes ocasiões de desenvolvimento da cultura da manga, pois podem indicar variação da importância de uma ou mais populações de plantas daninhas em diferentes estágios de desenvolvimento dessa cultura.

$\mathrm{Na}$ região norte de Minas Gerais diversos levantamentos fitossociológicos foram realizados (SARMENTO et al., 2015; SANTOS et al., 2015; BATISTA et al., 2016; BATISTA et al., 2017; SARMENTO et al., 2017) e mostraram-se pertinentes na identificação das espécies e podem, assim, ser extrapolados para as práticas de manejo de controle das plantas daninhas nas diversas culturas. Portanto, torna-se necessário realizar estudos com a cultura da manga para que os resultados possam subsidiar estratégias de manejo das espécies infestantes de forma eficiente.

Neste contexto, objetivou-se realizar o levantamento fitossociológico das espécies de plantas daninhas infestantes na cultura da mangueira aos dois e oito anos após plantio em Janaúba, norte de Minas Gerais.

\section{MATERIAL E MÉTODOS}

O levantamento fitossociológico das plantas daninhas foi realizado no mês de Abril de 2016 em áreas de produção de manga pertencentes à fazenda Califórnia na cidade de Janaúba, região norte do estado de Minas Gerais, com coordenadas geográficas de $15^{\circ} 82^{\text {ee }} 89^{\prime \prime}$ Sul e $43^{\circ} 26^{\text {ec}} 92^{\prime \prime}$ Oeste, altitude de $516 \mathrm{~m}$. O clima da região é do tipo "Aw" (tropical com inverno seco), com temperatura média anual de $25^{\circ} \mathrm{C}$ e precipitação média de $648 \mathrm{~mm}$ nos últimos dez anos (INMET, 2017). Os dados de temperatura e precipitação registrados no período de outubro de 2015 a abril de 2016 constam na Figura 1.

Foram avaliados dois pomares de mangueira 'Palmer' com 2 ha cultivados em solo de textura argilosa e irrigados via microaspersão. O primeiro pomar, com idade de dois anos de implantação, foi cultivado com mangueiras no espaçamento de $2 \mathrm{~m}$ entre plantas. Já o segundo pomar de manga, com oito anos de implantação, possuía espaçamento de quatro metros entre plantas. A distância entre as duas áreas era de aproximadamente $120 \mathrm{~m}$.

Para o levantamento da comunidade de plantas daninhas foi utilizado o método padrão do quadrado inventário $(0,5 \mathrm{~m}$ x $0,5 \mathrm{~m}$ ), sendo este lançado aleatoriamente uma vez na área útil de cada parcela, recolhendo todas as plantas, em caminhamento em zigue-zague (BRAUN-BLANQUET, 1979; ERASMO et al., 2004). As plantas daninhas foram coletadas com estrutura completa, com sistema radicular e parte aérea.

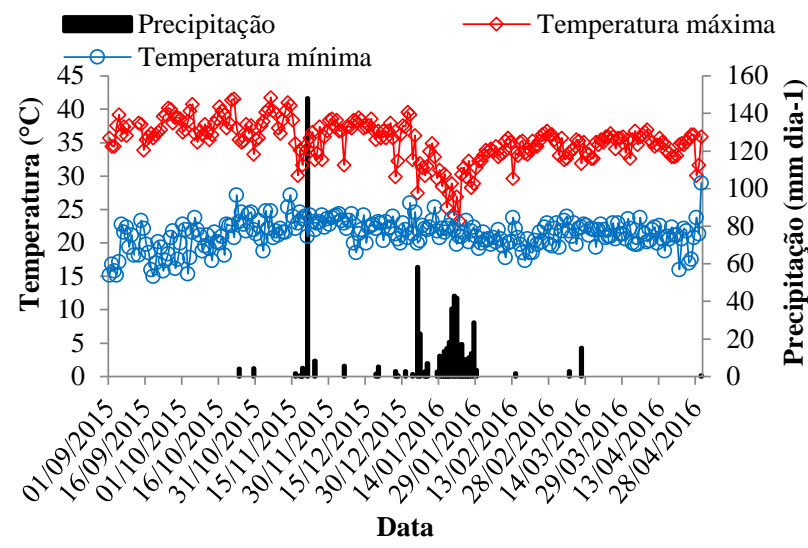

Figura 1. Temperatura máxima e mínima e precipitação durante o período de outubro de 2015 a abril de 2016 na área experimental. Figure 1. Maximum and minimum temperature and rainfall during the period from October 2015 to April 2016 in the experimental area.

Em cada amostra as plantas daninhas foram quantificadas e classificadas quanto à família, gênero e espécie (LORENZI, 2008). Logo após foram recolhidas e acondicionadas em sacos de papel e levadas para o laboratório, onde foram pesadas para obtenção do seu peso verde. Em seguida, foram colocadas em estufa de circulação de ar forçada, onde permaneceram por 72 horas a uma temperatura de $65{ }^{\circ} \mathrm{C}$, sendo depois novamente pesadas para obtenção do seu peso seco.

Com os dados da classificação e quantificação das espécies coletadas foi possível calcular as seguintes variáveis fitossociológicas: frequência $(\mathrm{F})$, que permite avaliar a distribuição das espécies na área; frequência relativa $(\mathrm{Fr})$; densidade (D), que possibilita determinar quantidade de plantas por espécie por unidade de área; densidade relativa (Dr); abundância (A), que indica sobre a concentração das espécies na área; abundância relativa (Ar); índice de valor de importância (IVI) e índice de valor de importância relativa (IVIr) (BRAUN-BLANQUET, 1979) que indicam a prioridade de controle de certa daninha em relação a outras; e índice de similaridade (IS) (SORENSEN, 1972) que indica a porcentagem de espécies comuns as duas áreas. As variáveis Fr, Dr e Ar possibilita obter informações sobre a relação de cada espécie com as outras espécies encontradas na área, sendo variáveis utilizadas para obtenção do IVI. Para os cálculos das variáveis foram utilizadas as seguintes fórmulas:

- Frequência $(\mathrm{F})$ :

$$
\mathrm{F}=\frac{\left(\mathrm{n}^{\circ} \text { de quadrados que contêm a espécie }\right)}{\left(\mathrm{n}^{\circ} \text { de quadrado obtidos (área total }\right)}
$$

- Frequência relativa $(\mathrm{Fr})$ :

$$
\mathrm{Fr}=\frac{(100 \mathrm{x} \text { Frequência da espécie })}{(\text { Frequência total de todas às espécies })}
$$

- Densidade (D):

$$
\mathrm{D}=\frac{\left(\mathrm{n}^{\circ} \text { total de indivíduos por espécie }\right)}{\left(\mathrm{n}^{\circ}\right. \text { de quadrado obtidos (área total) }}
$$


- Densidade relativa (Dr):

$$
\operatorname{Dr}=\frac{(100 \times \text { Densidade da espécie })}{(\text { Densidade total de todas às espécies })}
$$

- Abundância (A):

$$
A=\frac{\left(n^{\circ} \text { total de indivíduos por espécie }\right)}{\left(n^{\circ} \text { total de quadrados que contém a espécie }\right)}
$$

- Abundância Relativa (Ar):

$$
\mathrm{Ar}=\frac{(100 \times \text { Abundância da espécie })}{\text { (Abundância total de todas as espécies) }}
$$

- Índice de Valor de Importância (IVI):

$$
\mathrm{IVI}=\mathrm{Fr}+\mathrm{Dr}+\mathrm{Ar}
$$

- IVI Relativa (IVIr):

IVIr $=\frac{(100 x \text { Valor de importância da espécie })}{(\text { valor de importância total de todas as espécies })}$

- Índice de Similaridade (IS):

$$
\mathrm{IS}=\left(\frac{2 \mathrm{a}}{\mathrm{b}+\mathrm{c}}\right) \times 100
$$

em que: a é igual ao número de espécies comuns às duas áreas; b e c é igual número total de espécies nas duas áreas comparadas.

\section{RESULTADOS}

$\mathrm{Na}$ área com pomar de manga em produção, aos oito anos após plantio, foram encontradas 17 espécies, pertencentes a 14 gêneros e oito famílias, destacando-se as famílias Poaceae, Malvaceae e Fabaceae com cinco, quatro e três espécies respectivamente (Tabela 1).

As espécies que apresentaram maiores frequências, ou seja, que se encontraram mais distribuídas pela área, foram Sida rhombifolia (1), Aeschynomene denticulata (0,8) e Cynodon dactylon (0,5). Essas espécies também apresentaram as maiores densidades, que representa a quantidade de plantas por espécies por unidade de área, sendo que a $S$. rhombifolia se destacou como a espécie mais abundante $(6,8)$ indicando sua concentração nesta área (Tabela 2).

\begin{tabular}{|c|c|c|}
\hline \multirow{2}{*}{ Família } & \multicolumn{2}{|c|}{ Espécies } \\
\hline & Nome científico & Nome comum \\
\hline \multirow{5}{*}{ Poaceae } & $\begin{array}{l}\text { Brachiaria } \\
\text { plantaginea }\end{array}$ & $\begin{array}{c}\text { Capim- } \\
\text { Marmelada }\end{array}$ \\
\hline & Cynodon dactylon & Grama-seda \\
\hline & Digitaria horizontalis & Capim-Colchão \\
\hline & Sorghum halepense & $\begin{array}{c}\text { Capim } \\
\text { Massambará }\end{array}$ \\
\hline & Brachiaria mutica & Capim-Angola \\
\hline \multirow{4}{*}{ Malvaceae } & Sida rhombifolia & Guanxuma \\
\hline & Sida urens & $\begin{array}{l}\text { Guanxuma } \\
\text { Dourada }\end{array}$ \\
\hline & Waltheria douradinha & Douradinha \\
\hline & Sida cordifolia & Malva-Branca \\
\hline \multirow{3}{*}{ Fabaceae } & $\begin{array}{l}\text { Aeschynomene } \\
\text { denticulata }\end{array}$ & Angiquinho \\
\hline & $\begin{array}{l}\text { Macroptilium } \\
\text { atropurpureum }\end{array}$ & Siratro \\
\hline & Senna obtusifolia & Mata-Pasto \\
\hline Portulacaceae & Portulaca oleracea & Beldroega \\
\hline Asclepiadaceae & Calotropis procera & Algodão-de-Seda \\
\hline Euphorbiaceae & Euphorbia heterophylla & Leiteiro \\
\hline Asteraceae & Bidens pilosa & Picão-Preto \\
\hline Convolvulaceae & Ipomoea triloba & Corda-de-Viola \\
\hline
\end{tabular}

Tabela 1. Espécies e famílias das plantas daninhas encontradas na área de mangueira, em produção, com oito anos após plantio.

Table 1. Species and families of weeds found in the area in mango production with after planting eight years.

Com a soma dos dados referentes a Fr, Dr e $\mathrm{Ar}$ foi possível obter o índice de valor de importância (IVI) e a partir deste se obtém o índice de valor de importância relativo (IVIr). Pode-se observar que a espécie com maior índice de valor de importância relativo foi Sida rhombifolia $(27,8 \%)$ (Figura 2).

Tabela 2. Número de quadrados que contém a espécie (NQCE), numero de indivíduos por espécies (NI/E), frequência (F), frequência relativa (Fr), densidade (D), densidade relativa (Dr), abundância (A), abundância relativa (Ar) e matéria seca (MS) das plantas daninhas

\begin{tabular}{|c|c|c|c|c|c|c|c|c|c|}
\hline Espécies & NQCE & $\mathrm{NI} / \mathrm{E}$ & $\mathrm{F}$ & $\operatorname{Fr}(\%)$ & D & $\operatorname{Dr}(\%)$ & A & $\operatorname{Ar}(\%)$ & MS (g) \\
\hline Sida rhombifolia & 10 & 68 & 1 & 22,22 & 6,8 & 45,63 & 6,8 & 15,51 & 83,91 \\
\hline Brachiaria Plantaginea & 4 & 9 & 0,4 & 8,88 & 0,9 & 6,04 & 2,25 & 5,14 & 46,73 \\
\hline Sida urens & 1 & 1 & 0,1 & 2,23 & 0,1 & 0,67 & 1 & 2,29 & 1,55 \\
\hline Aeschynomene denticulata & 8 & 18 & 0,8 & 17,76 & 1,8 & 12,08 & 2,25 & 5,13 & 15,85 \\
\hline Macroptilium atropurpureum & 3 & 4 & 0,3 & 6,66 & 0,4 & 2,68 & 1,33 & 3,03 & 13,13 \\
\hline Cynodon dactylon & 5 & 16 & 0,5 & 11,11 & 1,6 & 10,73 & 3,2 & 7,3 & 29,61 \\
\hline Waltheria douradinha & 1 & 1 & 0,1 & 2,22 & 0,1 & 0,69 & 1 & 2,29 & 49,37 \\
\hline Sida cordifolia & 2 & 6 & 0,2 & 4,44 & 0,6 & 4,02 & 3 & 6,84 & 2,53 \\
\hline Portulaca oleracea & 2 & 3 & 0,2 & 4,44 & 0,3 & 2,01 & 1,5 & 3,42 & 6,63 \\
\hline Calotropis procera & 1 & 1 & 0,1 & 2,23 & 0,1 & 0,69 & 1 & 2,29 & 46,13 \\
\hline Digitaria horizontalis & 1 & 4 & 0,1 & 2,23 & 0,4 & 2,68 & 4 & 9,12 & 44,8 \\
\hline Euphorbia heterophylla & 1 & 2 & 0,1 & 2,23 & 0,2 & 1,34 & 2 & 4,57 & 2,32 \\
\hline Sorghum halepense & 1 & 4 & 0,1 & 2,23 & 0,4 & 2,68 & 4 & 9,12 & 62,2 \\
\hline Bidens pilosa & 1 & 1 & 0,1 & 2,23 & 0,1 & 0,69 & 1 & 2,29 & 6,44 \\
\hline Senna obtusifolia & 1 & 6 & 0,1 & 2,23 & 0,6 & 4,02 & 6 & 13,68 & 14,38 \\
\hline Ipomoea triloba & 1 & 2 & 0,1 & 2,23 & 0,2 & 1,34 & 2 & 4,56 & 8,67 \\
\hline Brachiaria mutica & 2 & 3 & 0,2 & 4,44 & 0,3 & 2,01 & 1,5 & 3,42 & 33,79 \\
\hline Total & 45 & 149 & 4,5 & 100 & 14,9 & 100 & 43,83 & 100 & 468,1 \\
\hline
\end{tabular}
encontradas na área de mangueira, em produção, com oito anos após plantio.

Table 1. Number of squares containing species (NSCS), number of individuals per species (NI/S), frequency (F), relative frequency (Rf), density (D), relative density (Rd), abundance relative (Ar) and dry matter (DM) of weeds found in the area under mango production after eight years of cultivation. 


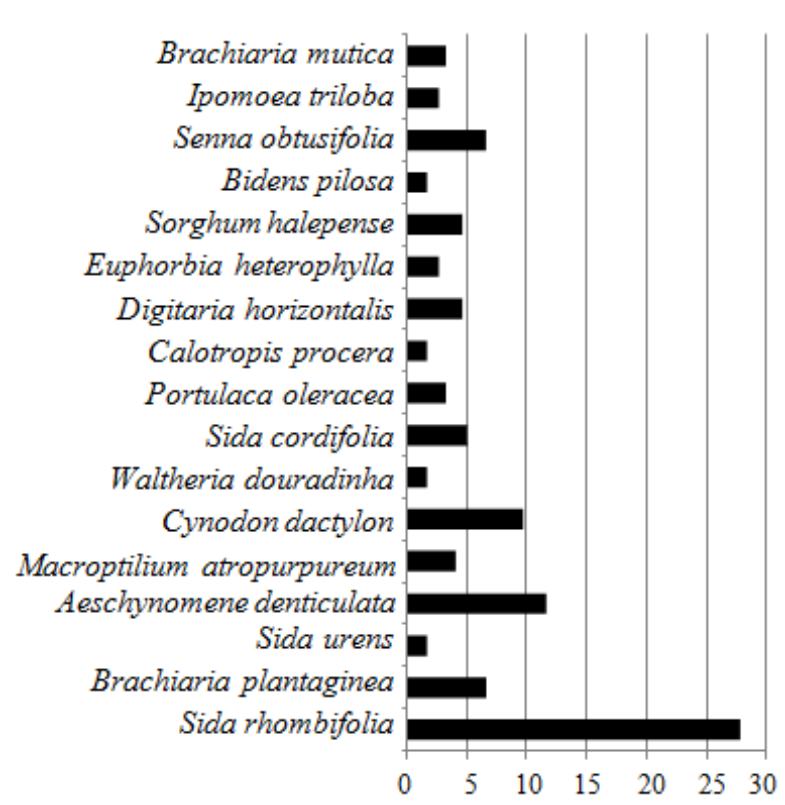

Figura 2. Índice de Valor de Importância Relativa das plantas daninhas na área de mangueira, em produção, com oito anos após plantio.

Figure 2. Relative Importance Value Index of weeds in the area in mango production with after planting eight years.
Na área com mangueira após dois anos de plantio foram encontradas cinco espécies, cada uma pertencente a um gênero diferente e quatro famílias, destacando-se a família Poaceae com duas espécies (Tabela 3).

Tabela 3. Espécies e famílias das plantas daninhas encontradas em área de mangueira após dois anos de plantio.

Table 3. Species and families of weeds found in mango area with after planting two years.

\begin{tabular}{lll}
\hline \multirow{2}{*}{ Família } & \multicolumn{2}{c}{ Espécies } \\
\cline { 2 - 3 } Poaceae & \multicolumn{1}{c}{ Nome Científico } & \multicolumn{1}{c}{ Nome comum } \\
\hline Paspalum urvillei & Sorghun halepense & Capim-da-Roça \\
\hline Fabaceae & Desmodium barbatum & Barbadinho \\
\hline Euphorbiaceae & Euphorbia irta & Erva-de-Santa-Luzia \\
\hline Convolvulaceae & Merremia macrocalyx & Corda-de-Viola \\
\hline
\end{tabular}

A espécie que apresentou maior frequência nesta área foi Paspalum urvillei $(0,8)$, (Tabela 4). Esta espécie também apresentou maior densidade $(7,7)$, sendo também a mais abundante $(9,63)$, apresentando o maior acúmulo de matéria seca e maior número de indivíduos por espécie (Tabela 4) e, além disso, apresenta maior índice de valor de importância relativo $(70,61)$ (Figura 3).

Tabela 4. Número de quadrados que contém a espécie (NQCE), número de indivíduos por espécies (NI/E), frequência (F), frequência relativa (Fr), densidade (D), densidade relativa (Dr), abundância (A), abundância relativa(Ar) e matéria seca (MS) das plantas daninhas encontradas em área de mangueira após dois anos de plantio.

Tabela 4. Number of squares containing species (NSCS), number of individuals per species (NI/S), frequency (F), relative frequency (Rf), density (D), relative density (Rd), abundance relative (Ar) and dry matter (DM) of weeds found in the area after two years of mango cultivation.

\begin{tabular}{lccccccccc}
\hline Espécies & NQCE & NI/E & F & Fr $(\%)$ & D & $\operatorname{Dr}(\%)$ & $\mathrm{A}$ & $\operatorname{Ar}(\%)$ & $\mathrm{MS}(\mathrm{g})$ \\
\hline Paspalum urvillei & 8 & 77 & 0,8 & 61,55 & 7,7 & 90,59 & 9,63 & 59,7 & 58,25 \\
Sorghum halepense & 2 & 3 & 0,2 & 15,35 & 0,3 & 3,53 & 1,5 & 9,3 & 32,3 \\
Desmodium barbatum & 1 & 2 & 0,1 & 7,7 & 0,2 & 2,35 & 2 & 12,4 & 27,72 \\
Euphorbia irta & 1 & 2 & 0,1 & 7,7 & 0,2 & 2,35 & 2 & 12,4 & 18,59 \\
Meremia macrocalix & 1 & 1 & 0,1 & 7,7 & 0,1 & 1,18 & 1 & 6,2 & 42,3 \\
\hline Total & 13 & 85 & 1,3 & 100 & 8,5 & 100 & 16,13 & 100 & 179,16 \\
\hline
\end{tabular}

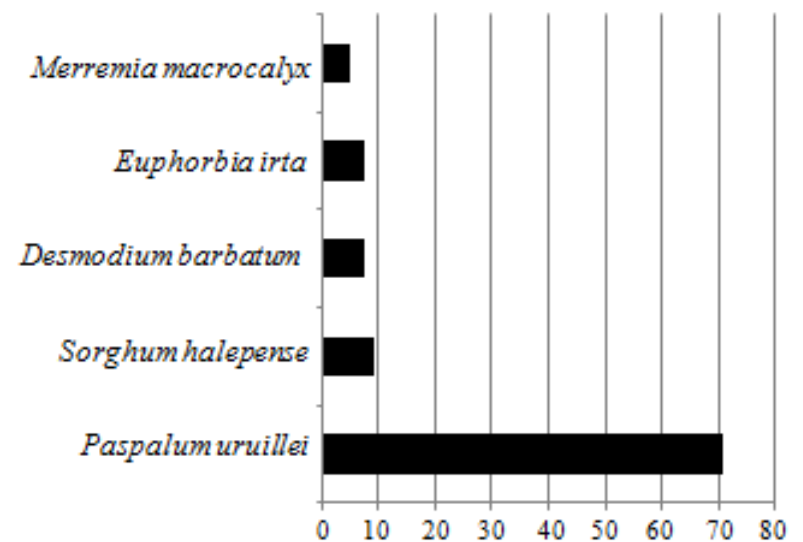

Figura 3. Índice de Valor de Importância Relativa das plantas daninhas em área de mangueira após dois anos de plantio.

Figure 3. Relative Importance Value Index of weeds in the area in after two years of mango cultivation.

\section{DISCUSSÃO}

Os resultados do presente estudo estão condizentes com levantamentos realizados em fruteiras onde a família poaceae se destacou em relação à área cultivada com mangueira, apresentando maior número de espécies (KIIL et al. 2001).
No pomar em produção, após oito de plantio, a $S$. rhombifolia se destacou como a espécie mais abundante. Esta espécie, conhecida popularmente por guanxuma ou vassourinha, é uma planta daninha encontrada em todas as regiões do país, infesta áreas de cultivos anuais, perenes e pastagens (SOUZA et al., 2011; BIANCO et al., 2014). Além da competição por recursos do ambiente, como água, luz e nutrientes, a guanxuma pode dificultar a colheita, por possuir caules muito lignificados e resistentes (BIANCO et al., 2014).

Assim, esta descrição torna-se importante do ponto de vista prático no controle da S. rhombifolia em pomares de mangueira, pois, a estratégia de manejo que será adotada, como controle o químico ou mecânico que são mais utilizaddos na região norte de Minas, devem ser realizados na fase inicial de crescimento lento desta espécie (BIANCO et al., 2014), antes que os caules lignifiquem.

Verificou-se ainda que da $S$. rhombifolia apresentou o maior IVIr (Figura 2). Este índice representa objetivamente quais espécies ou grupos de espécies devem ter maior prioridade na ordem de controle. Portanto, a S. rhombifolia deve receber prioridade máxima na ordem de controle de plantas daninhas na área em produção de mangueira. 
Os trabalhos já realizados com levantamento fitossociológico na cultura da mangueira não avaliaram o IVIr para as espécies encontradas (KIIL et al. 2001). Esta variável fitossociológica possibilita obter informações sobre a relação de cada espécie com as outras espécies encontradas nesta área, com isso é possível observar que S. rhombifolia se encontra mais distribuída, apresentando maior quantidade de plantas por espécie e maior acúmulo de matéria seca, sendo isso refletido em uma maior frequência, densidade e abundância (Tabela 2).

$\mathrm{Na}$ área após dois de implantação da mangueira foram encontradas diferentes e menor número de espécies em relação ao cultivo já em produção, com destaque para a espécie $d a$ família Poaceae. Salienta-se que á área foliar das plantas de manga com apenas dois anos de implantação permite que a radiação incida diretamente sob dossel de plantas daninhas em desenvolvimento. Portanto, como a avaliação foi realizada no período chuvoso da região, que associados às altas temperaturas (Figura 1) e intensa radiação solar favoreceram o desenvolvimento das plantas daninhas da família Poaceae, como Paspalum urvillei, que apresentam metabolismo fotossintético $\mathrm{C} 4 \mathrm{com}$ alta eficiência na utilização da luz solar. Já em condições de maior sombreamento, no caso da mangueira em produção após oito anos de implantação, as condições favorecem o predomínio de plantas daninhas que na sua maioria são espécies de folha larga com metabolismo C3, como a S. rhombifolia.

A P. urvillei é uma planta daninha de ciclo longo e extenso período de florescimento, tipicamente cespitosa, com elevado número de afilhos e com o envelhecimento, há um aumento progressivo na participação de colmos, bainhas foliares e panículas na biomassa aérea das plantas e, além disso, apresenta grau de lignificação em estádios avançados de florescimento (SCHEFFER-BASSO et al., 2002). Portanto, reforça a importância do controle na época adequada.

Esta espécie apresenta maior índice de valor de importância relativo (Figura 3) indicando que deve receber prioridade máxima na ordem de controle de plantas daninhas nesta área com dois anos de implantação.

A família Poaceae foi a que apresentou maior número de espécies tanto na área recém implantada quanto na área em produção, mas isto não significa dizer que as espécies daninhas pertencentes a esta família são as predominantes em áreas com cultivo da mangueira. Para isso ser confirmado é necessário realizar mais trabalhos em diferentes regiões e microrregiões, pois cada região e ecossistema têm sua peculiaridade quanto às plantas daninhas predominantes.

Confrontando os dados coletados, é possível observar que o número total de indivíduos por espécies na área em produção é de 149, enquanto na área recém implantada é de 85. O crescimento da mangueira é um pouco mais lento no seu período inicial de desenvolvimento, logo a incidência de plantas daninhas neste momento pode causar mais prejuízos a esta cultura, o que leva os produtores a utilizar, de forma mais intensiva, os métodos de controle como roçadas e controle químico. Isso pode ter contribuído para um número menor de indivíduos por espécies na área recém implantada em relação à área em produção.

$\mathrm{O}$ índice de similaridade das espécies encontradas nas áreas foi de 18,18\%, apresentando apenas duas espécies comuns às duas áreas, demonstrando que existe diferença entre as suas comunidades infestantes, visto que valores abaixo de $25 \%$ indicam pouca similaridade entre os fatores comparados por este índice (KUVA et al. 2007). Possivelmente, essa baixa similaridade entre as plantas daninhas presentes nos dois pomares de mangueira foi relacionada às questões das diferentes intensidades de sombreamento propiciado pela cultura aos dois de implantação e no pomar já em produção após oito anos implantado.

Salienta-se que essa informação é de extrema importância para o manejo das plantas daninhas no cultivo de mangueira em condições irrigadas do semiárido mineiro, pois, poderão otimizar às práticas de controle e, possivelmente, reduzir custos, já que os dois pomares apresentam diferentes espécies infestantes com características morfofisiológicas.

\section{CONCLUSÕES}

Paspalum urvillei e Sida rhombifolia são as principais espécies encontradas em pomares de mangueira após dois e oito anos após implantação, respectivamente, e devem receber prioridade máxima na ordem de controle de plantas daninhas.

Os pomares após dois e oito anos de implantação apresentam diferentes espécies infestantes comprovado pelo valor do índice de similaridade entre as duas áreas.

\section{REFERÊNCIAS}

BATISTA, P. S. C.; OLIVEIRA, V. S.; CAXITO, A. M.; CARVALHO, A. J.; ASPIAZÚ, I. Phytosociological survey of weeds in cultivars of common beans with different types of growth in the north of Minas Gerais. Planta Daninha, Viçosa, v. 34, n. 3, p. 497-507, 2016. DOI: http://dx.doi.org/10.1590/s0100-

83582016340300010

BATISTA, P. S. C.; OLIVEIRA, V. S.; SOUZA, V. B. de.; CARVALHO, A. J.; ASPIAZÚ, I. Phytosociological survey of weeds in erect prostrate cowpea cultivars. Planta Daninha, Viçosa, v. 35, n. 1, p. 1-9, 2017. DOI: http://dx.doi.org/10.1590/s0100-83582017350100031

BIANCO, S.; CARVALHO, L. B.; BIANCO, M.S. Crescimento e nutrição mineral de Sida rhombifolia. Planta Daninha, Viçosa, v. 32, n. 2, p. 311-317, 2014. DOI: http://dx.doi.org/10.1590/S010083582014000200008

BRAUN-BLANQUET, J. Fitossociologia: bases para el estudio de las comunidades vegetales. Madrid: H. Blume, 1979. 820 p.

CARNEIRO, M.A.; LIMA, A. M. N.; CAVALCANTE, I. H. L.; CUNHA, J. C.; RODRIGUES, M. S.; LESSA T. B. da S. Soil salinity and yield of mango fertigated with potassium sources. Revista Brasileira de Engenharia Agrícola e Ambiental, Campina Grande, v. 21, n .5, p. 310-316, 2017. DOI: http://dx.doi.org/10.1590/18071929/agriambi.v21n5p310-316

COTRIM, C. E.; COELHO, E. F.; SILVA, J. A.; SANTOS, M. R. dos. Irrigação com déficit controlado e produtividade de mangueira 'Tommy Atkins' sob gotejamento. Revista brasileira de agricultura irrigada, v. 11, n. 8 , p. 2229-2238, 2017. DOI: http://dx.doi.org/10.7127/RBAI.V11N800728

ERASMO, E. A. L.; PINHEIRO, L. L. A.; COSTA, N. V. Levantamento fitossociológico das comunidades de plantas infestantes em áreas de produção de arroz irrigado cultivado sob diferentes sistemas de manejo. Planta 
Daninha, Viçosa, v. 22, n. 2, p. 195-201, 2004. http://dx.doi.org/10.1590/S0100-83582004000200004

IBGE_INSTITUTO BRASILEIRO DE GEOGRAFIA E ESTATÍSTICA. Produção Agrícola Municipal. Sistema IBGE de Recuperação Automática-SIDRA. 2016. Disponível em: http:// http://www.sidra.ibge.gov.br. Acesso em: 14/09/2018.

INMET_INSTITUTO NACIONAL DE METEOROLOGIA. Banco de dados meteorológicos para ensino e pesquisa. 2016. Disponível em: http:// www.inmet.gov.br. Acesso em: 14/09/2018.

KILL, L. H. P.; LIMA, P. C. F.; LIMA, J. L. S. Plantas Invasoras em Frutíferas no Vale do SãoFrancisco. Petrolina: Embrapa Semi-Árido, 2001. 29 p. (Embrapa Semi-Árido. Documentos, 170).

KUVA, M. A.; PITELLI, R. A.; SALGADO, T. P.; ALVES, P. L. C. A. Fitossociologia de comunidades de plantas daninhas em agroecossistema cana-crua. Planta Daninha, Viçosa, v. 25, n. 3, p. 501-511, 2007. DOI: http://dx.doi.org/10.1590/S0100-83582007000300009

LORENZI, H. Plantas daninhas do Brasil: terrestres, aquáticas, parasitas e tóxicas. 4 ed. Nova Odessa: Instituto Plantarum, 2008. 640 p.

MEDEIROS, P. V. Q. de.; ANDRADE, G.; PEREIRA, R. G.; MENDONÇA, V.; NETO, J. F. Produção e qualidade de frutos de mangueira 'Tommy Atkins' adubadas com superfosfato simples no município de Assú-RN. Brazilian Journal of Applied Technology for Agricultural Science, Guarapuava, v. 7, n. 3, p. 07-16, 2014. DOI: http://dx.doi.org/10.5935/PAeT.V7.N3.01

OLIVEIRA, G. P.; SIQUEIRA, D. L. de.; SALOMÃO, L. C. C.; CECON, P. R.; MACHADO, D. L. M. Paclobutrazol and branch tip pruning on the flowering induction and quality of mango tree fruits. Pesquisa Agropecuária Tropical, Goiânia, v. 47, n. 1, p. 07-14, 2017. http://dx.doi.org/10.1590/1983-40632016v4743861

OLIVEIRA, M. B.; PEREIRA, M. C. T.; MIZOBUTSI, G. P.; MAIA. V. M.; SILVA, J. F.; OLIVEIRA, J. A. A.; COSTA, I. J. S.; NIETSCHE, S.; SANTOS, E. F.; MOUCO, M. A. C. Paclobutrazol and tip pruning in the management of 'Palmer' mango trees in the semi-arid region of Brazil. Acta horticulture, The Hague, v. 1, n. 1075, p. 149-156, 2015. DOI: https://dx.doi.org/10.17660/ActaHortic.2015.1075.16

PITELLI, R. A. Estudos fitossociológicos em comunidades infestantes de agroecossistemas. Jornal Conserb, v. 1, n. 2, p. 1-7, 2000.

SANTOS, M. R. dos; DONATO, S. L. R.; FARIA, L. N.; COELHO, E. F.; COTRIM JUNIOR, P. R. F. Tommy Atkins' mango yield and photosynthesis under water deficit in semiarid region of Bahia. Irrigation strategies with water deficit in 'Tommy Atkins' mango tree. Engenharia Agrícola, Jaboticabal, v. 36, n. 6, p. 10961109, 2016. DOI: http://dx.doi.org/10.1590/1809-4430eng.agric.v36n6p1096-1109/2016

SANTOS, R. C.; FERREIRA, E. A.; SANTOS, J. B.; OLIVEIRA, M. C.; SILVA, D. V.; PEREIRA, G. A. M.; GALON, L.; ASPIAZÚ, I.; MATTOS, N. P. Phytosociological characterization of weed species as affected by soil management. Australian Journal of Crop Science, v. 9, n. 2, p. 112-119, 2015.
SARMENTO, H. G. dos S.; CAMPOS FILHO, J. M.; ASPIAZÚ, I.; RODRIGUES, T. M.; FERREIRA, E. A. Levantamento fitossociológico de plantas daninhas em áreas de bananicultura no Vale do Rio Gorutuba, norte de Minas Gerais. Agro@mbiente On-line, Boa Vista, v. 9, n. 3, p. 308-316, 2015. DOI: http://dx.doi.org/10.18227/1982-8470ragro.v9i3.2314

SARMENTO, H. G. dos S.; RODRIGUES, T. M.; ASPIAZÚ, I.; CORSATO, C. E. Phytosociological survey in pineapple cultivated in northern Minas Gerais. Nativa, Sinop, v. 5, n. 4, p. 231-236, 2017. DOI: http://dx.doi.org/10.5935/2318-7670.v05n04a01

SARWAR, M.; HAMED, M.; YOUSAF, M.; HUSSAIN, M. Surveillance on population dynamics and fruits infestation of tephritid fruit flies (Diptera: Tephritidae) in mango (Mangifera indica L.) Orchards of Faisalabad, Pakistan. International Journal of Scientific Research in Environmental Sciences, v. 2, n. 4, p. 113-119, 2014. DOI: https://dx.doi.org/10.12983/ijsres-2014-p0113-0119

SCHEFFER-BASSO S. M.; RODRIGUES， G. L.; BORDIGNON, M. V. Caracterização Morfofisiológica e Anatômica de Paspalum urvillei (Steudel). Revista Brasileira de Zootecnia, Viçosa, v. 31, n. 4, p. 16741679, 2002. DOI: http://dx.doi.org/10.1590/S151635982002000700009

SILVA, D. J.; MOUCO, M. A. do C.; GAVA, C. A. T.; GIONGO, V.; PINTO, J. M. Composto orgânico em mangueiras (Mangifera indica L.) cultivadas no semiárido do nordeste brasileiro. Revista Brasileira de Fruticultura, Jaboticabal, v. 35, n. 3, p. 875-882, 2013. DOI: http://dx.doi.org/10.1590/S010029452013000300026

SHARMA, G.; KUMAR, N.; WEIR, B. S.; HYDE, K. D.; SHENOY, B. D. The ApMat marker can resolve Colletotrichum species: a case study with Mangifera indica. Fungal Diversity, v. 61, n. 1, p. 117-138, 2013. DOI: http://dx.doi.org/10.1007/s13225-013-0247-4

SORENSEN, T. A. Method of stablishing groups equal amplitude in plant society based on similarity of species content. In: ODUM, E. P. Ecologia. 3. ed. México: Interamericana, 1972. p. 341-405.

SOUZA, M. C.; PARREIRA, M. C.; AMARAL, C. L. do.; ALVES, P. L. da C. A. Efeito da época sobre a emergência de Sida rhombifolia e Solanum viarum em diferentes profundidades de semeadura. Revista Ceres, Viçosa, v. 58, n. 6, p. 749-754, 2011. DOI: http://dx.doi.org/10.1590/S0034-737X2011000600011

VASCONCELOS, M. da C. da C.; SILVA, A. S. A.; LIMA, R. S. Interferência de Plantas Daninhas sobre Plantas Cultivadas. Agropecuária Científica no Semiárido, Campina Grande, v. 8, n. 1, p. 01-06, 2012.

WEI, J.; LIU, G.; LIU, D.; CHEN, Y. Influence of irrigation during the growth stage on yield and quality in mango (Mangifera indica L). PLOS One, v. 12, n. 4, p. 01-10, 2017. https://dx.doi.org/10.1371/journal.pone.0174498 